established that there was poor access to hospice support for vulnerable people.

Aims To enable vulnerable people in North East Essex to live well with their life limiting illness and die with dignity in a place of their choice.

Methods A three-year project, funded by Macmillan was set up to facilitate the services needed to enable the aims to be reached. The focus was on those from the local homeless community, those living in areas of deprivation and those identified through the lung cancer early diagnosis scheme. As referrals increase from these groups, the future aim is to focus on networking with other support groups and improve hospice care for patients with mental illness, learning disabilities, LGBTQ+ groups and refugees.

Results

1. Successful liaisons with homeless support groups, neighbourhood schemes and services for vulnerable people in North East Essex;

2. Joint working between lung oncology services and hospice to improve the uptake of palliative support for patients with lung cancer;

3. A Macmillan information hub, staffed by volunteers, providing information to patients;

4. Referrals have increased by $51 \%$ in areas of deprivation due to improved communication/liaison with local support groups;

5. Patients have included homeless, drug and alcohol users, hoarders, patients with learning disabilities, mental health issues and those from ethnic minority groups.

Conclusion By providing information about the hospice services to the support groups and neighbourhood schemes local knowledge has improved. The service and referrals to the hospice for the most marginalised of our population have grown.

\section{P-29 COMMUNITY REPRESENTATION AND CULTURAL DIVERSITY OF THE MODERN-DAY HOSPICE}

Anne-Marie Bourke, Maxwell Charles. Marie Curie Hospice, Newcastle upon Tyne, UK

\subsection{6/bmjspcare-2019-HUKNC.53}

Background National guidance advocates that patients living with life limiting illnesses have access to individualised palliative care (General Medical Council, 2010). Evidence suggests that Black and Asian Minority Ethnic (BAME) groups are less likely than White patients to access palliative services (Markham, Islam \& Faull, 2014; Tackling Inequalities in End of Life Care for Minority Groups VCSE Health and Wellbeing Alliance Project Group, 2018). Negative consequences of this include patients experiencing symptoms and being denied the opportunity to consider advance care planning.

Aims The aim of this service evaluation was to establish the extent to which the documented ethnicity of patients referred to our hospice inpatient (IPU) and day therapies units (DTU) compare to the ethnic diversity of our local community (taken from the 2011 electoral ward census data).

Methods Documented ethnicity data of all patients admitted to the IPU or accepted onto the DTU caseload between November 2017 and December 2018 were retrospectively collected from SystmOne. Census data (2011) for the hospice electoral ward were obtained from the Office for National Statistics.
Results The populations of our IPU and DTU were not representative of the ethnic make-up of our locality during the study period. $47 \%$ of our local community identify as ethnicities other than White. In contrast, $90 \%$ of IPU admissions were White (2\% Asian/Asian British, 1\% Black/African/Caribbean/Black British and 7\% not recorded). Similarly, 91\% of people attending our DTU were White (2\% Asian/Asian British and $7 \%$ not recorded).

Conclusion The documented ethnicity of our service users does not reflect the ethnic diversity of our local populace. It is impossible to conclude from this project why ethnicities other than White are underrepresented. Potential barriers need to be investigated but might include a lack of awareness of the services we provide.

Since these data were collected, hospice staff have met with local religious leaders to demystify palliative care and establish if hospice services need to adapt to better serve our community.

\section{P-30 WHO IS HOSPICE MISSING?}

Giovanna Cruz, Diane Corrin, Cheryl Young, Lonan Oldam, Anne Mills, Sarah McGhee. Hospice Isle of Man, Douglas, Isle of Man

\subsection{6/bmjspcare-2019-HUKNC.54}

Background Hospice Isle of Man's aim is to provide access to those who could benefit regardless of diagnosis. The hospice began offering services to non-cancer patients in 2016. Little is known about how many could benefit or the extent of the hospice's involvement by disease category. The Isle of Man is not included in Hospice UK's PopNAT.

Aims To estimate the number of persons who could have potentially benefited from palliative and end-of-life care. To understand differences in hospice involvement by disease category.

Methods The Isle of Man Registry maintains weekly Excel sheets with death certificate data. We extracted variables of interest for the period between 1 January 2013 to 31 December 2018. We created a variable for palliative need based on cause of death where there was any mention of cancer, Alzheimer's/dementia, organ failure or a neurodegenerative condition. Using hospice records, we identified persons who received hospice care for the years 2016-2018. We estimated unmet need by comparing hospice involvement and presence of a condition likely to benefit from palliative care and compared hospice involvement by palliative disease category.

Results There were 5,005 deaths between 2013 and 2018, representing $1 \%$ of the population per year. Prior to 2016, the hospice cared for 30\% of persons who died. By 2018, $39 \%$ had hospice care representing a $21 \%$ increase from 2016. Of all deaths between 2013 and 2018, 30\% had a palliative condition and hospice care. An additional 34\% had a palliative condition but no hospice care. The hospice was involved in $88 \%$ of all cancer cases but only in a quarter or less of cases with organ failure (28\%), neurodegenerative conditions (26\%), or Alzheimer's/dementia (16\%).

Conclusions Since 2016, more persons received hospice care. However, persons with non-cancer conditions who could benefit remained under-represented compared to those with cancer. Future studies are needed to understand the factors contributing to this difference. 